\title{
Neoadjuvante Radiochemotherapie bei Speiseröhrenkrebs Standard?
}

\begin{abstract}
Den 5-Jahres-Ergebnissen von CROSS(ChemoRadiotherapy for Oesophageal cancer followed by Surgery Study) zufolge lebten Patienten mit Karzinomen von Speiseröhre oder ösophagogastralem Übergang länger, wenn sie zusätzlich zur chirurgischen Therapie eine neoadjuvante Radiochemotherapie erhalten hatten. Nun liegen Langzeitdaten vor.
\end{abstract}

nsgesamt 368 Patienten mit klinisch resezierbarem, lokal fortgeschrittenem Karzinom von Speiseröhre oder öso-

phagogastralem Übergang (Stadium T1 N1 M0 oder T2-3 N0-1 M0) waren randomisiert neoadjuvat mit Carbopla-

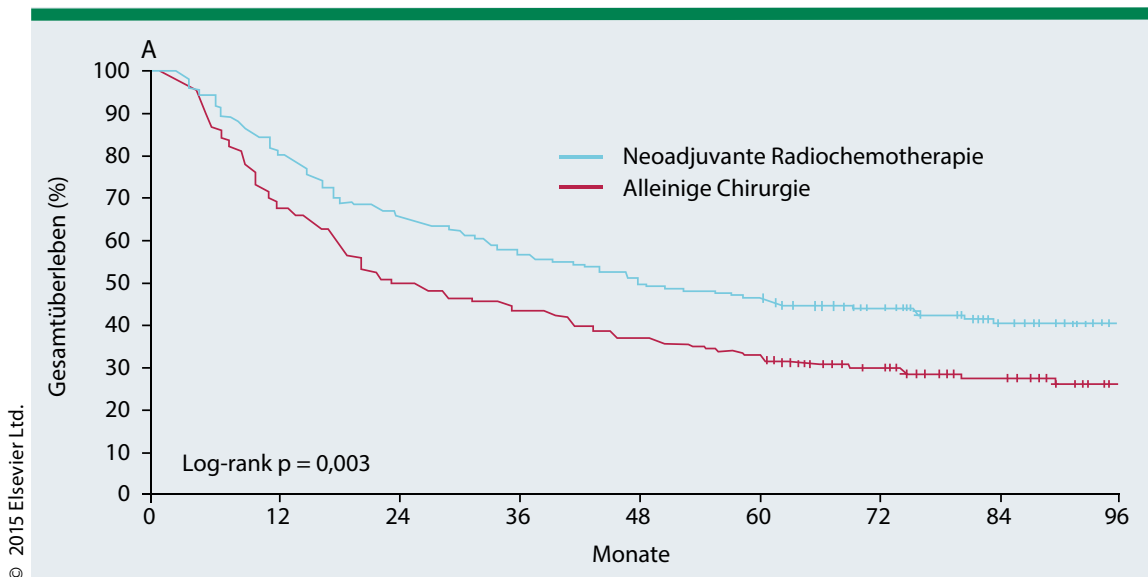

Abb. 1: Eine neoadjuvante Chemotherapie verbesserte das Überleben von Ösophaguskarzinompatienten signifikant. tin plus Paclitaxel sowie gleichzeitiger Bestrahlung (41,4 Gy) behandelt worden und hatten sich anschließend einer Operation unterzogen $(n=180)$ oder waren nur operiert worden $(n=188)$. Nach median 84,1 Monaten lag das Gesamtüberleben (OS) in der Gruppe mit neoadjuvanter Radiochemotherapie bei median 48,6 Monaten, in der Vergleichsgruppe bei median 24,0 Monaten (Hazard Ratio $[H R]$ 0,68; $\left.p_{\text {log-rank }}=0,003\right)($ Abb. 1).

Der Vorteil der neoadjuvanten Therapie zeigte sich besonders ausgeprägt bei Patienten mit Plattenepithelkarzinomen (81,6 vs. 21,1 Monate; HR 0,48; p $_{\text {log- }}$ rank $=0,008)$, war aber auch bei Patienten mit Adenokarzinom signifikant $(43,2$ vs. 27,1 Monate; HR 0,73; $\mathrm{p}_{\text {log-rank }}=0,038$ ).

Fazit: Beim resezierbaren, lokal fortgeschrittenen Karzinom von Ösophagus oder ösophagogastralem Übergang ist die neoadjuvante Radiochemotherapie vor Operation der Standard. Friederike Klein

Shapiro J et al. Neoadjuvant chemoradiotherapy plus surgery versus surgery alone for oesophageal or junctional cancer (CROSS): longterm results of a randomised controlled trial. Lancet Oncol. 2015;16(9):1090-8.

\section{Magenkarzinom: Individuellere Therapieentscheidungen in der Erstlinie}

\section{Beim fortgeschrittenen Magen- karzinom (GC) ist die Kombination aus dem Fluoropyrimidin S-1 und Cisplatin alle 5 Wochen (SP5) als ein Standard anzusehen. Jetzt wurde ein alternatives Schema (SP3) damit verglichen.}

n der Phase-III-Studie wurden chemotherapienaive Patienten mit einem metastasierten und/oder rezidivierten Karzinom des Magens oder eines Adenokarzinoms des gastroösophagealen Übergangs entweder mit SP alle $5(\mathrm{n}=309)$ oder alle $3(n=306)$ Wochen behandelt (SP5: 80-120 mg/d S-1 an den Tagen 1-21, Cisplatin $60 \mathrm{mg} / \mathrm{m}^{2}$ an den Tagen $1+8$, q5w und SP3: $80 \mathrm{mg} / \mathrm{m}^{2} / \mathrm{d} \mathrm{S}-1$ an den Ta- gen 1-14 und Cisplatin $60 \mathrm{mg} / \mathrm{m}^{2}$ an Tag 1, q3w). Ziel war es, die Nichtunterlegenheit bzw. Überlegenheit von SP3 zu prüfen. Im Median erhielten die Patienten im SP3-Arm 5 Zyklen, im SP5-Arm 3 Zyklen der jeweiligen Therapie.

Das progressionsfreie Überleben (PFS) unter SP3 erwies sich nach median 32,4 Monaten gegenüber SP5 sogar als länger (median 5,5 vs. 4,9 Monate; Hazard Ratio [HR] 0,82; p = 0,0418 für Überlegenheit). Der absolute, aber doch relativ geringe Unterschied im PFS übersetzte sich jedoch nicht in einen Überlebensvorteil (14,1 vs. 13,9 Monate; HR 0,99; $\mathrm{p}=0,9068)$. Bei Patienten mit messbarer Erkrankung lagen die Ansprechraten in beiden Studienarmen ähnlich hoch (60 vs. $50 \%$; p = 0,065).
Beide Regimes wurden gut toleriert, Anämien und Neutropenien $\geq$ Grad 3 traten jedoch häufiger unter SP3 auf (19 vs. $9 \%$ und 39 vs. $9 \%$ ).

Fazit: SP3 ist SP5 hinsichtlich des PFS überlegen und hinsichtlich des Gesamtüberlebens vergleichbar, jedoch mit etwas höherer Toxizität. Beide Strategien kommen als First-Line-Therapie für $\mathrm{Pa}$ tienten mit fortgeschrittenen GC infrage, die Auswahl sollte sich nach den individuellen Bedürfnissen des jeweiligen $\mathrm{Pa}$ tienten richten.

Friederike Klein

Ryu MH et al. Comparison of two different S-1 plus cisplatin dosing schedules as first-line chemotherapy for metastatic and/or recurrent gastric cancer: a multicenter, randomized phase III trial (SOS). Ann Oncol. 2015;26(10):2097-101. 